\title{
Randomised, placebo controlled trial of effect of a leukotriene receptor antagonist, montelukast, on tapering inhaled corticosteroids in asthmatic patients
}

\author{
Claes-Göran Löfdahl, Theodore F Reiss, Jonathan A Leff, Elliot Israel, Michael J Noonan, \\ Albert F Finn, Beth C Seidenberg, Thomas Capizzi, Sudeep Kundu, Philippe Godard
}

\section{Abstract}

Objective To determine the ability of montelukast, a leukotriene receptor antagonist, to allow tapering of inhaled corticosteroids in clinically stable asthmatic patients.

Design Double blind, randomised, placebo controlled, parallel group study. After a single blind placebo run in period, during which (at most) two inhaled corticosteroids dose decreases occurred, qualifying, clinically stable patients were allocated randomly to receive montelukast (10 $\mathrm{mg}$ tablet) or matching placebo once daily at bedtime for up to 12 weeks. Setting 23 academic asthma centres in United States, Canada, and Europe.

Participants 226 clinically stable patients with chronic asthma receiving high doses of inhaled corticosteroids (113 randomised to montelukast and 113 to placebo). Interventions Every 2 weeks, the inhaled corticosteroids dose was tapered, maintained, or increased (rescue) based on a standardised clinical score.

Main outcome measures Last tolerated dose of inhaled corticosteroids.

Results Compared with placebo, montelukast allowed significant $(\mathrm{P}=0.046)$ reduction in the inhaled corticosteroid dose (montelukast $47 \% v$ placebo $30 \%$; least square mean difference $17.6 \%, 95 \%$ confidence interval 0.3 to 34.8 ). Fewer patients on montelukast $(18(16 \%)$ v $34(30 \%)$ placebo, $\mathrm{P}=0.01)$ required discontinuation because of failed rescue.

Conclusions Montelukast reduces the need for inhaled corticosteroids among patients requiring moderate to high doses of corticosteroid to maintain asthma control.

\section{Introduction}

People with persistent asthma often require daily treatment to control symptoms, usually with inhaled corticosteroids. ${ }^{1}$ Recent publications have shown that in patients whose asthma is not completely controlled by inhaled corticosteroids, adding a second drug rather than increasing the dose of corticosteroid achieves better control. ${ }^{23}$ Leukotriene receptor antagonists have complementary effects to corticosteroids on inflammatory events in asthma, ${ }^{45}$ providing a basis for these additive clinical effects. Montelukast is a potent, specific, cysteinyl leukotriene receptor antagonist which, given daily, improves asthma control in adults ${ }^{67}$ and children, ${ }^{8}$ protects against exercise induced bronchoconstriction, ${ }^{9}$ and decreases sputum eosinophil counts. ${ }^{10}$ Subgroup analyses of previously reported clinical trials have suggested that montelukast has an additive effect with inhaled corticosteroids. ${ }^{611} 12$
We performed a placebo controlled, double blind, randomised trial to investigate whether daily treatment with montelukast $(10 \mathrm{mg}$ ) allowed reduction of inhaled corticosteroids while maintaining asthma control.

\section{Participants and methods}

\section{Participants}

We recruited 226 non-smoking adults with a clinical history of asthma for at least 1 year. Their forced expiratory volume in 1 second had to be $\geqslant 70 \%$ of the predicted value (after withholding $\beta$ agonist for $\geqslant 6$ hours) at the prestudy visit and to improve by $\geqslant 15 \%$ (absolute value) after inhaled $\beta$ agonist. Patients were excluded if they had had emergency treatment for an asthma exacerbation within 1 month, been in hospital for asthma within 3 months, or had an unresolved upper respiratory tract infection within 3 weeks of the prestudy visit. Patients must have been treated with a stable, twice daily dose of inhaled corticosteroids for at least 3 weeks before the prestudy visit.

The protocol was approved by the ethics review committees or institutional review boards of all participating centres. Written informed consent was obtained from all participants.

\section{Study design}

The study was conducted at 23 academic medical centres or large group practices in the United States, Canada, and Europe. We used a randomised, double blind, placebo controlled, two period, parallel group design to investigate the effect of montelukast $(10 \mathrm{mg}$ tablet once daily at bedtime, irrespective of food) on the ability to taper the dose of inhaled corticosteroids over 12 weeks. Patients were recruited over 3 months. A prestudy visit preceded a single blind, placebo run in period, which lasted at most 7 weeks. The purpose of the run in was to achieve or approach the minimum inhaled corticosteroid dose necessary to maintain clinical stability. After, at most, two dose reductions, clinically stable patients were eligible for randomisation if they demonstrated during the 7-10 days before or at the allocation visit a forced expiratory volume in 1 second $\geqslant 90 \%$ of the run in baseline value, less than pre-established levels of asthma symptoms and $\beta$ agonist use, peak flow at least $65 \%$ of the maximum, and required a prespecified minimum dose of inhaled corticosteroids. Allocation was according to a computer generated allocation schedule with a blocking factor of 4 . Three blocks were initially sent to each centre. Patients remained on their randomised treatment (10 mg montelukast or placebo) throughout the study. Follow up was stopped at the end of 12 weeks' treatment.

\section{University Hospital, Lund, Sweden Claes-Göran Löfdahl, professor of respiratory medicine Department of Pulmonary and Immunology, Merck Research Laboratories, Rahway, NJ 07065 , USA \\ Theodore F Reiss, senior director Jonathan A Leff, associate director Beth C Seidenberg, vice president, clinical research \\ Department of Biostatistic and Research Data System, Merck Research Laboratories Thomas Capizzi, senior director, clinical biostatistics \\ Sudeep Kundu, senior biometrician \\ Brigham and Women's Hospital, Boston, MA, USA \\ Elliot Israel, director, clinical research \\ continued over}

BMJ 1999;319:87-90

website

extra

The members of the Montelukast Study Group are listed on the BMJ's website

www.bmj.com 
Allergy Associate PC Research Center, Portland, OR, USA clinical investigator

Allergy and Asthma Centers of

Charleston, North

Charleston, SC,

USA

Albert F Finn,

clinical associate

professor of medicine, microbiology, and immunology

Arnaud de

Vilenueve Hospital,

Montpellier, France

Philippe Godard,

professor of medicine

Correspondence to:

T F Reiss

Theodore Reiss@

Merck.com
Michael J Noonan,

\section{Methods}

Participants returned to the clinic every 2 weeks, and their inhaled corticosteroids dose was adjusted (decreased, increased, or maintained) based on a previously validated composite clinical score (box). ${ }^{13}$ The dose of inhaled corticosteroids was tapered or increased by about $25 \%$ (according to pre-established criteria). Unstable patients who failed to regain clinical stability after an increase in dose of corticosteroids were withdrawn from the trial and classified as "failed rescue." Patients were also withdrawn from the study if they interrupted their study drug for more than 5 consecutive days or had worsening asthma requiring treatment with oral, intravenous, or intramuscular corticosteroids.

Diary cards-As part of the taper criteria, patients recorded daytime symptoms, ${ }^{14}$ "as needed" use of $\beta$ agonists, and morning and afternoon peak expiratory flow on a daily diary card.

Spirometry-Standard equipment and quality control procedures were used across all study sites. Participants performed at least three forced vital capacity manoeuvres achieving American Thoracic Society standards. ${ }^{15}$ The best forced expiratory volume was recorded from each set of measurements.

\section{Statistical analysis}

The primary analysis was by intention to treat. All patients with postallocation data were included in the analysis. The last tolerated dose of inhaled corticosteroids was the primary end point and was defined as the last dose during the treatment period at which the subject had a composite clinical score of 2 or 3. An endpoint committee, blind to subject allocation, determined all last tolerated doses for all allocated patients based on pre-established criteria. This end point was

\section{Criteria for tapering dose of inhaled corticosteroids: composite} clinical score

Before $\beta$ agonist $\mathrm{FEV}_{1} \geqslant 90 \%$ of value at

allocation

Daytime symptom score $\leqslant 120 \%$ of

Yes $=1$ point $\quad \mathrm{No}=0$ points

preallocation* baseline

$\beta$ Agonist use $\leqslant 135 \%$ of preallocation*

baseline

Yes $=1$ point $\quad \mathrm{No}=0$ points

Yes $=1$ point $\quad$ No $=0$ points

Score

3 Points: patient stable $=$ taper inhaled corticosteroid

2 Points: patient stable $=$ maintain dose of inhaled corticosteroid

0 or 1 point: patient unstable $=$ increase dose of inhaled corticosteroid

*Established 7-10 days before allocation visit to clinic

Table 1 Characteristics of patients before randomisation. Values are mean (SD) unless stated otherwise

\begin{tabular}{lcc} 
Variable & Placebo & Montelukast \\
\hline Sample size* & 113 & 113 \\
\hline Mean (range) age (years) & $41(16-68)$ & $40(17-70)$ \\
\hline No of men & 61 & 47 \\
\hline Duration of asthma (years) & $19(14.0)$ & $18(13.3)$ \\
\hline FEV ${ }_{1}(\%$ predicted) & $82.3(12.9)$ & $84.8(11.1)$ \\
\hline Daytime symptom score† & $1.38(0.8)$ & $1.29(0.7)$ \\
\hline$\beta$ Agonist use (puffs/day) & $2.8(2.6)$ & $2.4(2.2)$
\end{tabular}

* Includes all patients randomly allocated in period two. One patient in the montelukast treatment group did not have any postallocation data available. $†$ Daily average of four questions, $0-6$ point scale. $\mathrm{FEV}_{1}=$ forced expiratory volume in 1 second. compared between treatment groups as the percentage change from preallocation baseline (to adequately summarise changes in corticosteroids of different potencies) with an analysis of variance model. This model was used to construct $95 \%$ confidence intervals for the least square means and differences of means. All statistical tests were two tailed, and a $P$ value $\geqslant 0.05$ was considered significant.

The number (percentage) of patients with failed rescue and tapering were prespecified as a secondary end point and were compared between treatment groups by the Cochran-Mantel-Haenszel test. The time to failed rescue was compared with a log rank test.

The study was designed with a sample size of 100 patients in each group to have $90 \%$ power to detect $(\alpha=0.050$, two tailed test) a mean difference between the two treatment groups of about 30 percentage points in the percentage change from baseline in dose of inhaled corticosteroid. ${ }^{13}$

\section{Results}

\section{Randomisation and withdrawals}

Of the 226 patients (113 randomised to placebo, 113 to montelukast), one could not be included in the analysis (montelukast) because of insufficient postallocation data. A further 48 patients did not complete 12 weeks' treatment: 31 in the placebo group (16 for "failed rescue," nine for clinical adverse experiences, three for protocol deviations, and three withdrew consent) and 17 in the montelukast group (seven for "failed rescue," four for clinical adverse experiences, four for protocol deviations, one withdrew consent, and one lost to follow up). The two treatment groups did not differ in baseline values (table 1).

The mean inhaled corticosteroid dose for the 226 allocated patients was reduced $37.3 \%$ before randomisation (tables 2 and 3). The preallocation doses of inhaled corticosteroids, daily symptoms, $\beta$ agonist use, and forced expiratory volumes were comparable between treatment groups (tables 1 and 3). Compared with placebo, montelukast significantly $(\mathrm{P}=0.046)$ reduced the last tolerated dose of inhaled corticosteroids. Mean percentage changes from preallocation baseline were $47 \%$ and $30 \%$ for the montelukast and placebo groups, respectively (least square mean difference $17.6 \%, 95 \%$ confidence interval 0.3 to 34.8 ; table 3). Tapering was consistent across all inhaled corticosteroids.

Forty five $(40 \%)$ patients on montelukast and 33 $(29 \%)$ on placebo tapered completely off inhaled corticosteroids. Seventy (62\%) patients on montelukast compared with $57(50 \%)$ on placebo had their dose tapered by $50 \%$ or more of the prerandomisation baseline; $31(28 \%)$ on montelukast and $41(36 \%)$ on placebo could not taper at all $(\mathrm{P}=0.055)$.

Thirty four $(30 \%)$ patients on placebo had failed rescues compared with $18(16 \%)$ on montelukast $(\mathrm{P}=0.01)$. In addition, time to failed rescue was better with montelukast compared with placebo $(\mathrm{P}=0.042$, $\log$ rank test).

No significant differences occurred between treatment groups in the change in spirometry measurements (\% change in forced expiratory volume 2.7\% montelukast $v 5.4 \%$ placebo), asthma symptom score 
(0.07 $v 0.12)$, and $\beta$ agonist use (0.29 $v 0.36$ puffs/day) at the last tolerated dose.

There were no significant differences in the frequency of clinical or laboratory adverse experiences between placebo and montelukast patients. Nine patients on placebo and four on montelukast stopped the trial because of adverse effects (worsening asthma for all montelukast patients).

\section{Discussion}

We found that, compared with placebo, montelukast (10 $\mathrm{mg}$, administered once daily at bedtime) allowed reduction of moderate to high doses of inhaled corticosteroids while maintaining clinical stability in patients with chronic asthma. These data demonstrate that montelukast has an additive benefit to inhaled corticosteroids in this setting. Another leukotriene receptor antagonist, pranlukast, has been shown to decrease requirements for inhaled corticosteroids, although the study used a different design. ${ }^{16}$ Given the potential limitations of prolonged exposure to high doses of inhaled corticosteroids, ${ }^{17-20}$ therapeutic strategies providing clinical control of asthma with the lowest corticosteroid dose would be valuable and are consistent with present treatment guidelines. ${ }^{1}$

Patients often receive higher doses of inhaled corticosteroids than required. ${ }^{17}$ For example, during the placebo run in period of this trial, patients reduced their inhaled corticosteroids dose by about $37 \%$, consistent with previous publications. ${ }^{21}$ Additionally, a pilot study for our trial that carefully evaluated the taper and rescue protocols also found appreciable reductions of inhaled corticosteroids. Because we expected a large placebo response we included a 5-7 week, single blind, placebo run in period.

At allocation, patients appeared well matched. Although the inhaled corticosteroids dose was slightly lower in montelukast patients, the similarity in forced expiratory volume, symptoms, and use of $\beta$ agonists supports similar disease severity in these patients. Furthermore, a lower inhaled corticosteroid dose at allocation might provide less opportunity for tapering in montelukast patients.

A second, important finding of this study was that the number of failed rescues was lower with montelukast than placebo. Patients taking montelukast were more likely to be successfully rescued after an episode of worsening asthma and therefore more likely to remain clinically stable.

Since the study was time based (12 weeks after randomisation) not event based, it had certain limitations.

\section{Key messages}

- Leukotriene receptor antagonists have complementary action to inhaled corticosteroids in asthma

- Many patients receive higher doses of inhaled corticosteroids than clinically required

- In this placebo controlled trial, montelukast allowed significant reduction of inhaled corticosteroid doses

- Fewer patients receiving montelukast had failed rescue than patients receiving placebo
Table 2 Mean (range) doses* of inhaled corticosteroids before study and before allocation

\begin{tabular}{|c|c|c|}
\hline & Prestudy ( $\mu \mathrm{g} / \mathrm{day})$ & Preallocation ( $\mu \mathrm{g} / \mathrm{day})$ \\
\hline \multicolumn{3}{|l|}{ Fluticasone: } \\
\hline $\begin{array}{l}\text { Placebo } \\
\end{array}$ & 1000.0 (500 to 1500 ) & 714.3 (300 to 1500 ) \\
\hline Montelukast & 1156.3 (500 to 2000 ) & 687.5 (300 to 1500$)$ \\
\hline \multicolumn{3}{|l|}{ Beclomethasone: } \\
\hline Placebo & 1253.3 (800 to 2000 ) & 753.3 (500 to 1500 ) \\
\hline Montelukast & 1165.0 (800 to 2000 ) & 715.0 (400 to 1500 ) \\
\hline \multicolumn{3}{|l|}{ Budesonide: } \\
\hline Placebo & 1288.9 (800 to 2400 ) & 770.4 (400 to 1600 ) \\
\hline Montelukast & 1078.3 (800 to 2400 ) & 591.3 (400 to 1200 ) \\
\hline \multicolumn{3}{|l|}{ Flunisolide: } \\
\hline Placebo & 2027.8 (1500 to 3000 ) & 1333.3 (500 to 3000 ) \\
\hline Montelukast & 1911.8 (1500 to 3000 ) & 1264.7 (500 to 3000 ) \\
\hline \multicolumn{3}{|l|}{ Triamcinolone: } \\
\hline Placebo & 2017.4 (1200 to 3200$)$ & 1321.7 (400 to 2400 ) \\
\hline Montelukast & 1991.1 (1200 to 3600 ) & 1217.8 (400 to 2400 ) \\
\hline
\end{tabular}

${ }^{*}$ Dose actuated, not delivered at mouthpiece.

Table 3 Mean (SD) changes in inhaled corticosteroid dose ( $\mu \mathrm{g} /$ day) during study

\begin{tabular}{lcccc} 
Treatment group & Prestudy & Pre-allocation & Last tolerated & $\begin{array}{c}\% \text { reduction from } \\
\text { allocation }\end{array}$ \\
\hline Placebo & $1681(670)$ & $1079(557)$ & $727(743)$ & $30 \%$ \\
\hline Montelukast & $1588(646)$ & $976(553)$ & $526(716)$ & $47 \%{ }^{*}$ \\
\hline
\end{tabular}

${ }^{*} \mathrm{P}=0.046$ compared with placebo.

In some patients, tapering (based on clinical stability) could have continued beyond 12 weeks; therefore, the true number of patients who could have stopped inhaled corticosteroids is unknown. Additionally, since patients were not followed beyond the 12 weeks we do not know how many required the reintroduction of inhaled corticosteroids to maintain clinical stability. A study with longer follow up would allow more complete understanding of the clinical importance of the effect of montelukast observed in this study.

Montelukast was generally well tolerated in this asthmatic population taking high doses of inhaled corticosteroids. Double blind, placebo controlled, clinical trials have confirmed the short term ( $\leqslant 3$ month) safety profile in adults and children (ages 6-14)..$^{6-9}$ Additional long term clinical trials and real world clinical experience are necessary to understand the long term safety profile of leukotriene receptor antagonists.

In conclusion, our results suggest that leukotriene receptor antagonists such as montelukast may be useful for long term treatment of asthmatic patients requiring high doses of inhaled corticosteroids.

The members of the Montelukast Study Group are listed on the $B M J$ s website. We thank Ji Zhang for expert statistical advice and Lars-Olof Eriksson for study monitoring.

Contributors: The manuscript was written collaboratively by TFR, JAL, C-GL, EI, PG, and SK. TFR, JAL, BCS, SK, C-GL, and TC participated in the design of the clinical trial. C-GL, EI, MJN, AFF, and PG participated in data collection. SK and TC participated in the statistical planning and analysis. TFR is the study guarantor.

Funding: This study was supported by a grant from Merck Research Laboratories, Rahway, NJ.

Competing interests: C-GL is a member of a Merck advisory board, AFF owns shares of Merck stock and is a member of the Merck speakers bureau, MJN is a member of Merck speakers bureau and a member of a scientific council sponsored by Merck, and EI is a member of a scientific council sponsored by Merck.

1 US Department of Health and Human Services. National Heart, Lung, and Blood Institute/World Health Organisation Workshop Report. Global initiative 
for asthma: global strategy for asthma management and prevention. Bethesda, MD: National Heart, Lung, and Blood Institute, National Institutes of Health, 1995. (Publication No 95-3659, chapter 7.)

2 Pauwels RA, Lofdahl CG, Postma DS, Tattersfield AE, O'Byrne P, Barnes PJ, et al for the Formoterol and Corticosteroids Establishing Therapy (FACET) International Study Group. Effect of inhaled formoterol and (FACEI) International Study Group. Effect of inhaled formoterol and

3 Greening AP, Ind PW, Northfield M, Shaw G, on behalf of Allen and Hanburys Limited UK Study Group. Added salmeterol versus higher-dose corticosteroid in asthma patients with symptoms on existing inhaled corticosteroid. Lancet 1994;344:219-24.

4 Dworski RT, Fitzgerald GA, Oates JA, Sheller JR. Effect of oral prednisone on airway inflammatory mediators in atopic asthma. Am J Respir Crit Care Med 1994:149:953-9.

5 Rididick CA, Ring WL, Baker JR, Hodulik CR, Bigby TD. Dexamethasone increases expression of 5-lipoxygenase and its activating protein in human monocytes and THP-1 cells. Eur J Biochem 1997;246:112-8.

6 Reiss TF, Chervinsky P, Dockhorn RJ, Shingo S, Seidenberg BC, Edwards TB for the Montelukast Clinical Research Study Group. Montelukast, a once-daily leukotriene receptor antagonist, in the treatment of chronic asthma. Arch Intern Med 1998;158:1213-20.

7 Noonan MJ, Chervinsky P, Brandon M, Zhang J, Kundu S, McBurney J, et al for the Montelukast Asthma Study Group. Montelukast, a potent leukotriene receptor antagonist, causes dose-related improvements in chronic asthma. Eur Respir J 1998;11:1232-9.

8 Knorr B, Matz J, Bernstein JA, Nguyen H, Seidenberg BC, Reiss TF, et al. Montelukast for chronic asthma in 6- to 14-year-old children. a randomized, double-blind trial. JAMA 1998;279:1181-6.

9 Leff JA, Busse WW, Pearlman D, Bronsky EA, Kemp J, Hendeles L, et al. Treatment of mild asthma and exercise-induced bronchoconstriction with montelukast, a leukotriene receptor antagonist. $N$ Engl J Med 1998;339:147-52

10 Pizzichini E, Leff JA, Reiss TF, Hendeles L, Boulet LP, Wei LX, et al. Montelukast reduces airway eosinophilic inflammation in asthma: a randomized, controlled trial. Eur Respir J (in press).
11 Reiss TF, Sorkness CA, Stricker W, Botto A, Busse WW, Kundu S, et al. Effects of montelukast (MK-0476), a potent cysteinyl leukotriene receptor antagonist, on bronchodilation in asthmatic patients treated with and without inhaled corticosteroids. Thorax 1997;52:45-8.

12 Reiss TF, Altman LC, Chervinsky P, Bewtra A, Stricker WE, Noonan GP, et al. Effects of montelukast (MK-0476), a new potent cysteinyl leukotriene $\left(\mathrm{LTD}_{4}\right)$ receptor antagonist, in patients with chronic asthma. J Allergy Clin Immunol 1996;98:528-34.

13 Kundu S, Noonan N, Friedman BS, Reiss TF, Leff JA. Use of a composite clinical score allows safe tapering of inhaled corticosteroids in asthmatic patients. Am J Respir Crit Care Med 1997;155:A352.

14 Santanello NC, Barber BL, Reiss TF, Friedman BS, Juniper EF, Zhang J. Measurement characteristics of two asthma symptom diary scales for use in clinical trials. Eur Respir J 1997;10:646-51.

15 American Thoracic Society. Standardization of spirometry. Am J Respir Crit Care Med 1995;152:1107-36.

16 Tamaoki J, Kondo M, Sakaj N, Nakata J, Takemura H, Nagai A, et al on behalf of the Tokyo Joshi-Idai Asthma Research Group. Leukotriene antagonist prevents exacerbation of asthma during reduction of high-dose inhaled corticosteroid. Am J Respir Crit Care Med 1997; 155:1235-40.

17 Barnes PJ, Pedersen S, Busse WW. Efficacy and safety of inhaled corticosteroids. New developments. Am J Respir Crit Care Med 1998;157:S1-53.

18 Cumming RG, Mitchell P, Leeder SR. Use of inhaled corticosteroids and the risk of cataracts. N Engl J Med 1997;337:8-14.

19 Hanania NA, Chapman KR, Kesten S. Adverse effects of inhaled corticosteroids. Am J Med 1995;98:196-208.

20 Kamada AK, Szefler SJ, Martin RJ, Boushey HA, Chinchilli VM, Drazen JM, et al. Issues in the use of inhaled glucocorticoids. Am J Respir Crit Care Med 1996:153:1739-48.

21 Erzurum SE, Leff JA, Cochran JE, Ackerson LM, Szefler SJ, Martin RJ, et al. Lack of benefit of methotrexate in severe, steroid-dependent asthma. Ann Int Med 1991;114:353-60.

(Accepted 19 April 1999)

\section{What are leukotrienes and how do they work in asthma?}

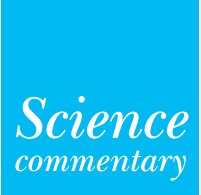

Acute asthma attacks are often triggered by allergens or exercise. Inflammatory molecules called leukotrienes are one of several substances which are released by mast cells during an asthma attack, and it is leukotrienes which are primarily responsible for the bronchoconstriction. In chronic, more severe cases of asthma, general bronchial hyperreactivity (or smooth muscle twitchiness) is largely caused by eosinophils, which are attracted into the bronchioles by leukotrienes (and other chemoattractants) and which themselves also produce leukotrienes. Thus leukotrienes seem to be critical both in triggering acute asthma attacks and in causing longer term hypersensitivity of the airways in chronic asthma.

Leukotrienes are derived from arachidonic acid, the precursor of prostaglandins. There are two families of leukotrienes. The first group acts primarily in conditions in which inflammation is dependent on neutrophils, such as cystic fibrosis, inflammatory bowel disease, and psoriasis. The second group (cysteinylleukotrienes) is concerned primarily with eosinophil and mast cell induced bronchoconstriction in asthma. They bind to highly selective receptors on bronchial smooth muscle and other airway tissue (Annals of Internal Medicine 1997;127:472-80).

Drugs have now been designed which can interfere with the activity of leukotrienes. Both leukotriene synthesis inhibitors and cysteinylleukotriene receptor antagonists have recently been shown to protect asthmatic patients against asthma attacks, but they are not useful as "rescue remedies" once an attack has already started. They act by preventing leukotriene release from mast cells and eosinophils or by blocking the specific leukotriene receptors on bronchial tissues, thus preventing bronchoconstriction, mucus secretion, and oedema.
These drugs also reduce the influx of eosinophils, thus limiting inflammatory damage in the airway. These oral, non-steroidal, anti-inflammatory drugs reduce the incidence of acute asthma attacks when taken regularly.

Abi Berger, science editor, BMJ

\section{Corrections and clarifications}

Will the NHS pay awards help recruitment? In this editorial by Stephen Machin (10 April, p 958) it was stated that the government had accepted in full the recommendations of the pay review bodies. In fact, it declined to implement the recommendation of the Doctors' and Dentists' Review Body that an extra $£ 50 \mathrm{~m}$ should be paid to consultants from the year 2000 to compensate them for their substantially increased workload.

Guidelines for evaluating papers on educational interventions

In this article by the Education Group for Guidelines on Evaluation (8 May, pp 1265-7) the name of Jean Ker was spelt incorrectly in the list of contributors (p 1267).

Opposition to the Icelandic database is based on false information

The last sentence of this letter by Gisli Ragnarsson (15 May, p 1354) should have read: "The legislation simply forbids such use, and it is made impossible through encryption [not ecryption]."

Minerva

In her review of a case-control study showing the health benefits of exercise (15 May, p 1362)

Minerva wrongly ascribed the study to the Annals of Internal Medicine. The correct reference is Archives of Internal Medicine (1999;159:686-90). 